Copyright 2001 American Association of Physics Teachers. This article may be downloaded for personal use only. Any other use requires prior permission of the author and the American Association of Physics Teachers

\title{
Analysis of standing vertical jumps using a force platform
}

\author{
Nicholas P. Linthorne ${ }^{\text {a) }}$ \\ School of Exercise and Sport Science, The University of Sydney, Sydney, New South Wales, Australia
}

(Received 9 March 2001; accepted 8 May 2001)

\begin{abstract}
A force platform analysis of vertical jumping provides an engaging demonstration of the kinematics and dynamics of onedimensional motion. The height of the jump may be calculated (1) from the flight time of the jump, (2) by applying the impulse-momentum relation to the force-time curve, and (3) by applying the work-energy relation to the forcedisplacement curve.
\end{abstract}

\section{INTRODUCTION}

A force platform can be an excellent teaching aid in undergraduate physics classes and laboratories. Recently, Cross $^{1}$ showed how to increase student interest and understanding of elementary mechanics by using a force platform to study everyday human movements such as walking, running, and jumping. My experiences with using a force platform in undergraduate classes have also been highly favourable. The aim of this article is to show how a force platform analysis of the standing vertical jump may be used in teaching the kinematics and dynamics of one-dimensional motion. I use computer software that produces curves of velocity and displacement of the jumper's center of mass by numerical integration of the force-time record from a force platform. A simultaneous examination of these curves gives an effective illustration of the relations between the forces acting on a body and the resulting acceleration, velocity, and displacement of the body.

The curves obtained from the force platform may also be used to calculate the height of the jump, and three methods are presented here. The most straight-forward method is to determine the time spent in the airborne phase and then use the kinematic equations for one-dimensional motion under constant acceleration. A more accurate method of determining the jump height is to apply the impulse-momentum relation to the force-time record, and this provides an interesting example of numerical integration. The jump height may also be calculated by applying the work-energy theorem to the forcedisplacement curve, again using numerical integration.

\section{FORCE PLATFORM}

Force platforms have a wide range of applications, including automobile crash tests, clinical gait analysis, and sports technique analysis. A force platform is a rectangular metal plate, usually about $0.4 \mathrm{~m} \times 0.6 \mathrm{~m}$, with piezoelectric or strain gauge transducers attached at each corner to give an electrical output that is proportional to the force on the plate. Commercial force platform systems for analyzing human movement cost $\$ 10000-\$ 30000$, but with relatively modest expertise a Department of Physics could construct one for about $\$ 5000 .{ }^{1,2}$ A force platform measures the force exerted on it by the subject, and according to Newton's third law of motion this also gives the force exerted by the platform on the subject. In terrestrial human movement, the force exerted by the platform on the body is commonly called the 'ground reaction force'.

The jumps discussed in this article were recorded using a Kistler force platform that was set in concrete in the floor of our teaching laboratory. ${ }^{3}$ The vertical component of the ground reaction force of the jumper was sampled at $1000 \mathrm{~Hz}$ and recorded by an IBM compatible PC with a Windows 95 operating system. Data acquisition and analysis of the jumps were performed using a custom computer program (JUMP ANALYSIS) that was written using LABVIEW virtual instrument software.

\section{STANDING VERTICAL JUMPS}

Figure 1 shows the motions involved in a 'countermovement jump' and in a 'squat jump'. In a countermovement jump, the jumper starts from an upright standing position, makes a preliminary downward movement by flexing at the knees and hips, then immediately and vigorously extends the knees and hips again to jump vertically up off the ground. A countermovement jump is an example of a movement that benefits from the 'stretch-shorten cycle'. Many human movements such as running, jumping, and throwing involve muscle actions in which the desired motion is preceded by a movement in the opposite direction. The muscles are said to be 'pre-stretched' before shortening in the desired direction. Experiments have demonstrated that a prestretch enhances the force production and work output of the muscles in the subsequent movement. ${ }^{4}$ 


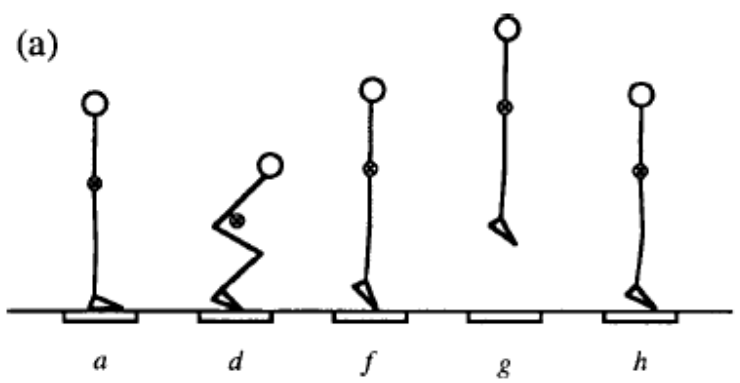

(b)

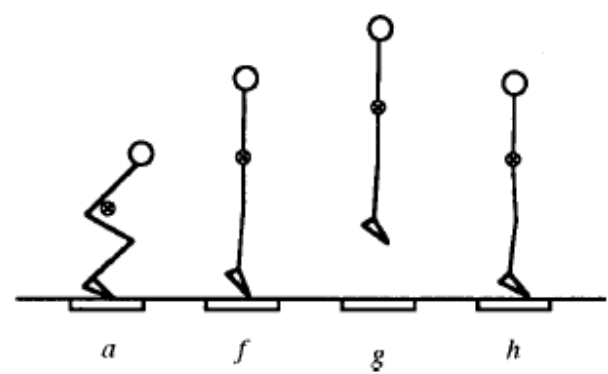

Fig. 1. Sequence of actions (a) in a countermovement jump and (b) in a squat jump. The jumper's center of mass (indicated by $\otimes$ ) moves strictly in the vertical direction. The jumper is shown at key times during the jump, as indicated by the letters. See the Appendix for an explanation of the key times. 
In a squat jump, the jumper starts from a stationary semisquatted position then vigorously extends the knees and hips to jump vertically up off the ground [Fig. 1(b)]. The jumper does not employ a preliminary downward phase (i.e., a countermovement) and so the jump does not involve prestretching of muscles. A squat jump is a slightly artificial movement that is rarely used in practice. The only example that comes to mind is the takeoff phase in ski-jumping. The countermovement jump is a much more natural jumping movement and most people can jump several centimeters higher in a countermovement jump than in a squat jump.

In my introductory mechanics classes, a skilled jumper is asked to perform countermovement jumps and squat jumps with maximum effort while standing on the force platform. Standing vertical jumps may be performed with and without the aid of armswing. In the jumps examined here, the jumper kept his hands on his hips throughout the movement.

\section{FORCE, ACCELERATION, VELOCITY, AND DISPLACEMENT}

During a vertical jump, the jumper must overcome body weight, and the resultant force acting on the jumper's center of mass (c.m.) is $F_{\mathrm{GRF}}-m g$, where $F_{\mathrm{GRF}}$ is the ground reaction force acting on the jumper, $m$ is the jumper's mass, and $g$ is the acceleration due to gravity. Curves of force-time, acceleration-time, velocity-time, displacement-time, and force-displacement are calculated from the ground reaction force obtained from the force platform. The time record of the resultant force acting on the jumper's c.m. is calculated by subtracting the jumper's body weight from the ground reaction force record. The velocity-time record is obtained by dividing the resultant force-time record by the jumper's body mass to give the acceleration-time record, and then numerically integrating with respect to time using the trapezoid rule. The displacement-time record is obtained by numerically integrating the velocity-time record, again using the trapezoid rule. Higher order integration procedures such as the Simpson rule do not improve the precision of the calculated parameters significantly. ${ }^{5}$

The integration calculations require that the velocity and vertical height of the jumper's c.m. be known at some instant. I use the start position of the jump, where the velocity of the jumper's c.m. is zero and the vertical height is set to zero. In the JUMP ANALYSIS program, the start of the jump is selected by moving a cursor along the force-time curve. It must be stressed that the velocity and displacement calculations are very sensitive to the initial conditions. For accurate calculations there must be a short period before commencing the jump where the jumper is completely motionless. This ensures that the ground reaction force at the start of the jump is precisely equal to the jumper's body weight and that the initial velocity is zero. It is not difficult to remain motionless in the standing position before the start of a countermovement jump. However, in a squat jump the jumper commences from a crouched position, and some jumpers require considerable practice to ensure that they produce a period of no motion before commencing the upward phase of the jump and that there is no initial downward motion.

Figures 2(a)-2(e) show curves of force-time, accelerationtime, velocity-time, displacement-time, and forcedisplacement for a countermovement jump. In the JUMP ANALYSIS program, a cursor may be moved along the forcetime curve and the corresponding points on the other curves are indicated as the cursor is moved. Although vertical jumping is a relatively simple movement skill, the physics of the vertical jump is not immediately obvious. Students find a simultaneous examination of the curves to be an illuminating exercise. The students carefully trace the evolution of the jump while identifying the key times and phases. They are asked to describe the actions of the jumper and note the relations between the force acting on the jumper and the resulting acceleration, velocity, and displacement of the jumper's c.m. The key times and phases during the jump are indicated on Figs. 2(a)-2(e) by the letters $a$ to $h$, and these are discussed in the Appendix.

Figure 3 shows a squat jump performed with the same vertical pushoff range as in the countermovement jump. That is, the height of the jumper's c.m. at the start of the squat jump was the same as the height of the c.m. at the lowest point of the countermovement jump. The physics of the squat jump is similar to that of the countermovement jump, and so the curves relating to the squat jump are not discussed here.

\section{CALCULATING THE HEIGHT OF A JUMP}

Before the jumper performs the jumps on the force platform, I ask the students to directly measure the 'height of the jump'. Most students decide that a good measure is the difference between the height of the jumper's c.m. when standing upright and the height of the c.m. at the peak of the jump. However, it is difficult to locate the jumper's c.m. and to monitor its motion during the jump. The students then realise that the rise of the jumper's heels during the jump is very nearly the same as the rise of the jumper's c.m. (see Fig. 1). A measurement of the rise of the jumper's heels is readily performed with a meter rule and a keen eye. ${ }^{6}$ This direct jump height measurement provides a baseline for the indirect measurements obtained later from the force platform.

Performance in a standing vertical jump may also be characterised by the flight height, which is the difference between the height of the c.m. at the peak of the jump and the height of the c.m. at the instant of takeoff. The flight height is slightly less than the rise relative to the height of the c.m. when standing upright because the jumper has the ankles extended at the instant of takeoff (see Fig. 1). Three methods of calculating the flight height from the force platform data are presented here; (1) the flight time method, (2) the impulse-momentum method, and (3) the work-energy method. ${ }^{7}$ All three methods calculate the flight height from knowledge of the velocity of the jumper's c.m. at the instant of takeoff. The relation between flight height and takeoff velocity may be obtained by applying the law of conservation of mechanical energy to the flight phase of the jump. In vertical jumping the effect of air resistance is negligible and so in the flight phase the jumper may be considered as a projectile in free flight. We consider the changes in kinetic energy and gravitational potential energy between the instant of takeoff (to) and the instant the jumper reaches the peak of the jump (peak):

$$
1 / 2 m v_{\text {to }}^{2}+m g y_{\text {to }}=1 / 2 m v_{\text {peak }}^{2}+m g y_{\text {peak }} \text {. }
$$

The vertical velocity at the peak of the jump is zero $\left(v_{\text {peak }}=0\right)$, and so the flight height is given by

$$
y_{\text {flight }}=y_{\text {peak }}-y_{\text {to }}=\frac{v_{\text {to }}{ }^{2}}{2 g} \text {. }
$$

where $v_{\text {to }}$ is the vertical takeoff velocity. 

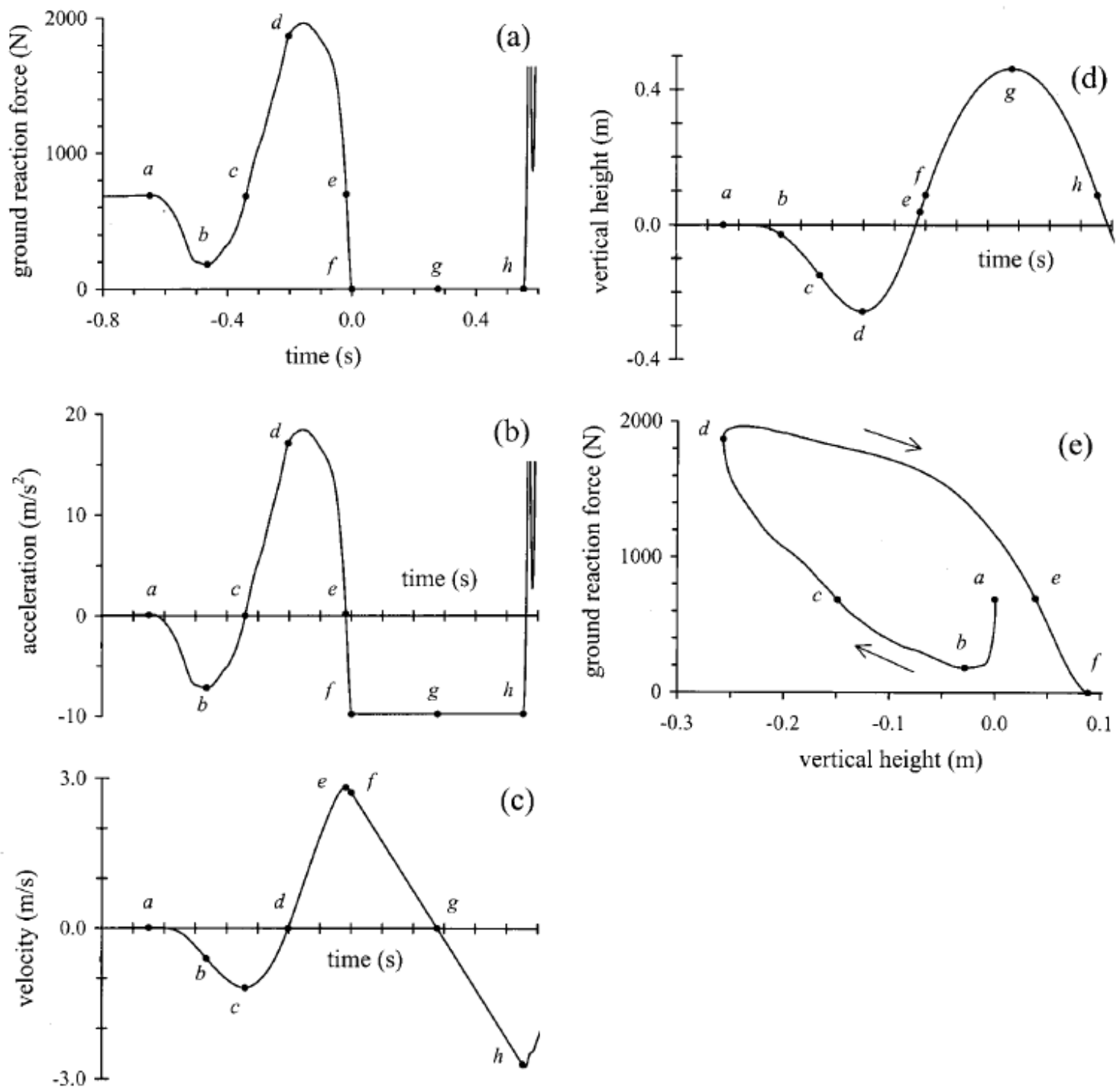

Fig. 2. Dynamic and kinematic curves for a countermovement jump: (a) ground reaction force curve, (b) acceleration-time curve, (c) velocity-time curve, (d) displacement-time curve, and (e) force-displacement curve. The time $t=0$ has been set to the instant of takeoff. The significance of the points indicated by the letters next to the curve is discussed in the Appendix. In the force-displacement curve, the flight and landing phases of the jump $(f-h)$ are not shown.

\section{A. Flight time method}

For a jumper in free flight, $v_{\mathrm{f}}-v_{\mathrm{i}}=-g\left(t_{\mathrm{f}}-t_{\mathrm{i}}\right)$, where $v_{\mathrm{i}}$ and $v_{\mathrm{f}}$ are the velocities at some initial and final times, $t_{\mathrm{i}}$ and $t_{\mathrm{f}}$. Here, we let the initial time be the instant of takeoff and the final time be the instant of landing. If we assume the height of the jumper's c.m. at the instant of landing is the same as at the instant of takeoff, then $v_{\mathrm{f}}=-v_{\mathrm{i}}$, and so the takeoff velocity of the jumper is given by

$$
v_{\text {to }}=\frac{g t_{\text {flight }}}{2},
$$

where $t_{\text {flight }}$ is the time of flight from the instant of takeoff to the instant of landing.
In the JUMP ANALYSIS program, the times of the takeoff and landing (points $f$ and $h$ ) are selected by moving two cursors on the force-time curve. For the countermovement jump shown in Fig. $2, t_{\text {flight }}=0.553 \mathrm{~s}$, and so $v_{\text {to }}=2.71 \mathrm{~m} / \mathrm{s}$ and $y_{\text {flight }}=37.5$ $\mathrm{cm}$. The squat jump shown in Fig. 3 has a shorter flight time $\left(t_{\text {flight }}=0.524 \mathrm{~s}\right)$ than the countermovement jump, and hence a $3.8 \mathrm{~cm}$ lower flight height $\left(y_{\text {flight }}=33.7 \mathrm{~cm}\right)$.

A jumper does not always have exactly the same body configuration at the instant of landing as at the instant of takeoff. The ankle and knee joints are usually almost fully extended at takeoff, but usually less so at landing. When vertical jumps are performed with the hands on the hips, the height of the jumper's c.m. at landing is usually about $1-4 \mathrm{~cm}$ lower than at takeoff and so the flight time method overestimates the true flight height by $0.5-2 \mathrm{~cm}^{5}$ This 
inaccuracy becomes more pronounced if the jumper employs armswing during the jump. At takeoff, the arms swing up in front of the face, but are then lowered slightly in preparation for the landing. The jumper therefore has an even greater height difference between takeoff and landing than when the hands remain on the hips.
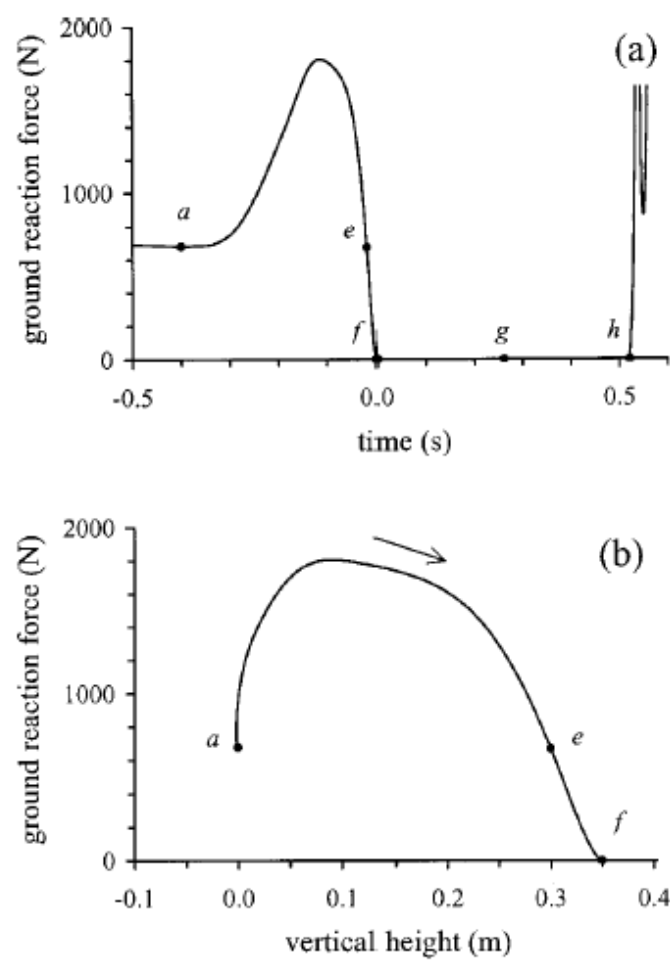

Fig. 3. Force curves for a squat jump: (a) ground reaction force curve and (b) force-displacement curve. The time $t=0$ has been set to the instant of takeoff. The key times $b, c$, and $e$ do not occur in a squat jump. In the force-displacement curve, the flight and landing phases of the jump $(f-h)$ are not shown.

\section{B. Impulse-momentum method}

The integral of a force over time (i.e., impulse) produces a change in the momentum of a body, $\mathbf{J}=\int \mathbf{F} d t=\Delta \mathbf{p}$. Applying the impulse-momentum theorem to the ground contact phase of the jump, starting from when the jumper is stationary $\left(v_{\mathrm{i}}=0\right.$ at $t_{\mathrm{i}}$ ) through to the instant of takeoff (at $t_{\mathrm{to}}$ ) gives:

$$
\int_{t_{\mathrm{i}}}^{t_{\mathrm{to}}}\left(F_{\mathrm{GRF}}-m g\right) d t=m v_{\mathrm{to}} .
$$

The impulse due to the resultant force on the jumper may be considered as two separate impulses:

$$
\int_{t_{\mathrm{i}}}^{t_{\mathrm{to}}} F_{\mathrm{GRF}} d t-\int_{t_{\mathrm{i}}}^{t_{\mathrm{to}}} m g d t=J_{\mathrm{GRF}}-J_{\mathrm{BW}}=m v_{\mathrm{to}},
$$

where $J_{\mathrm{GRF}}$ is the impulse due to the ground reaction force and $J_{\mathrm{BW}}$ is the impulse due to the jumper's body weight.
The impulses $J_{\mathrm{GRF}}$ and $J_{\mathrm{BW}}$ for the countermovement jump are shown in Fig. 4. In the JUMP ANALYSIS program, the initial and final times for the integration (points $a$ and $f$ ) are selected by moving two cursors on the force-time curve. The ground reaction force impulse is determined by calculating the area under the force-time curve by numerical integration. The body weight impulse is calculated from the reading of the ground reaction force curve of the first cursor. Precise selection of the initial time is irrelevant to the calculation of the resultant impulse, as long as the initial time is when the jumper is stationary before the start of the jump.

For the countermovement jump shown in Fig. $4, J_{\mathrm{GRF}}-J_{\mathrm{BW}}$ $=188 \mathrm{~N} \cdot \mathrm{s}$, and so $v_{\text {to }}=2.71 \mathrm{~m} / \mathrm{s}$ and $y_{\text {flight }}=37.5 \mathrm{~cm}$. The squat jump shown in Fig. 3 has a smaller resultant impulse $\left(J_{\mathrm{GRF}}-J_{\mathrm{BW}}=181 \mathrm{~N} \cdot \mathrm{s}\right)$ than the countermovement jump, and hence a lower flight height $\left(y_{\text {flight }}=33.7 \mathrm{~cm}\right)$.

For this jumper, the flight heights calculated using the flight time method are in excellent agreement with those calculated using the impulse-momentum method. It must be emphasised that the jumper in this study is not a typical jumper in that the height of the jumper's c.m. at landing was within a few millimeters of the height at takeoff [Fig. 2(d)]. The flight height calculated using the flight time method is usually a couple of centimeters greater than that calculated using the impulse-momentum method because the jumper is usually lower at landing than at takeoff.

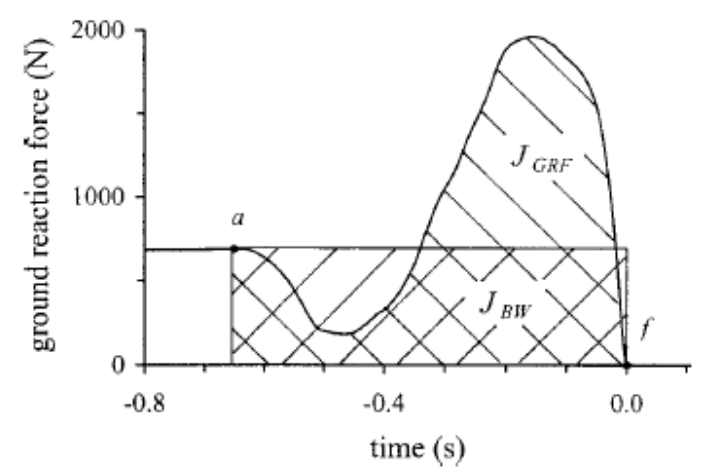

Fig. 4. Impulses due to the ground reaction force, $J_{\mathrm{GRF}}$, and due to the jumper's body weight, $J_{\mathrm{BW}}$, for the countermovement jump shown in Fig. 2.

\section{Work-energy method}

The integral of a force over displacement (i.e., work) produces a change in the kinetic energy of a body, $W=\int \mathbf{F} \cdot d \mathbf{r}=$ $\Delta \mathrm{KE}$. Applying the work-energy theorem to the ground contact phase of the jump, starting from when the jumper is stationary $\left(v_{\mathrm{i}}=0\right.$ at $\left.y=y_{\mathrm{i}}\right)$ through to the instant of takeoff (at $y$ $\left.=y_{\mathrm{to}}\right)$ gives

$$
\int_{y_{\mathrm{i}}}^{y_{\mathrm{to}}}\left(F_{\mathrm{GRF}}-m g\right) d y=1 / 2 m v_{\mathrm{to}}{ }^{2} .
$$

The resultant work may be considered as the work done by each of the two forces acting on the jumper: 


$$
\int_{y_{\mathrm{i}}}^{y_{\mathrm{to}}} F_{\mathrm{GRF}} d y-\int_{y_{\mathrm{i}}}^{y_{\mathrm{to}}} m g d y=W_{\mathrm{GRF}}-W_{\mathrm{BW}}=1 / 2 m v_{\mathrm{to}}{ }^{2},
$$

where $W_{\mathrm{GRF}}$ is the work done on the jumper by the ground reaction force and $W_{\mathrm{BW}}$ is the work done on the jumper by gravity.

The total work performed during the jump may be calculated starting from point $a$ (the start of the downward phase of the jump) or from point $d$ (where the jumper is again stationary at the lowest point of the countermovement). I prefer to use $d$, rather than $a$, as the start point in the work calculations as it is easier to understand the signs of the areas in the integration calculations. In the JUMP ANALYSIS program, the initial and final times for the integration (points $d$ and $f$ ) are selected by moving two cursors on the force-displacement curve. The work done by the ground reaction force is determined by calculating the area under the forcedisplacement curve by numerical integration, and the work done by the jumper's body weight is calculated from the reading of the ground reaction force curve for the first cursor. Figure 5 shows the work done by the two forces acting on the jumper, $W_{\mathrm{GRF}}$ and $W_{\mathrm{BW}}$.

For the countermovement jump shown in Fig. 5, $W_{\mathrm{GRF}}-$ $W_{\mathrm{BW}}=496 \mathrm{~J}-237 \mathrm{~J}=259 \mathrm{~J}$, and so $v_{\mathrm{to}}=2.73 \mathrm{~m} / \mathrm{s}$ and $y_{\text {flight }}=$ $38.0 \mathrm{~cm}$. The squat jump shown in Fig. 3 has a smaller resultant work $\left(W_{\mathrm{GRF}}-W_{\mathrm{BW}}=470 \mathrm{~J}-238 \mathrm{~J}=232 \mathrm{~J}\right)$ than the countermovement jump, and hence a lower flight height $\left(y_{\text {flight }}\right.$ $=33.5 \mathrm{~cm}$ ). An interesting exercise for the student is to use the two cursors to confirm that the resultant work performed between $a$ and $d\left(W_{\mathrm{a}-\mathrm{d}}\right)$ is zero, and that $W_{\mathrm{a}-\mathrm{c}}=-W_{\mathrm{c}-\mathrm{d}}$.

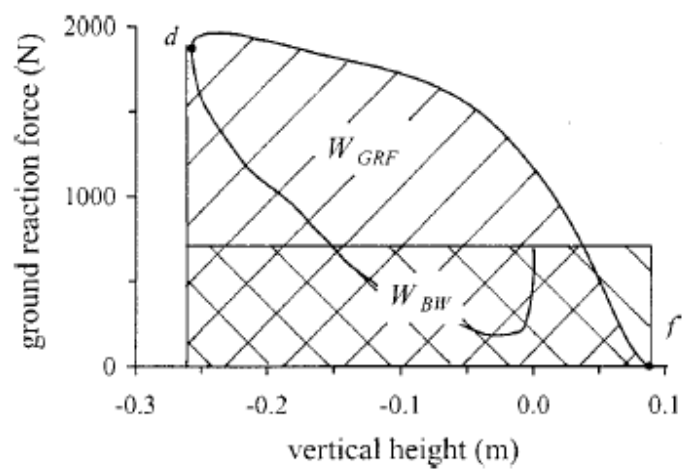

Fig. 5. Work done by the ground reaction force, $W_{\mathrm{GRF}}$, and by the jumper's body weight, $W_{\mathrm{BW}}$, for the countermovement jump shown in Fig. 2.

\section{Comparison of methods}

Of the three methods of calculating the flight height presented here, the flight time method is the simplest to perform. ${ }^{8}$ However, the flight time method usually overestimates the true flight height because the height of the jumper's c.m. at landing is lower than that at takeoff. The impulse-momentum method gives the most accurate calculation of flight height. In this method, the accuracy of the calculation is determined by correct selection of an instant before the start of the jump where the jumper is stationary and the ground reaction force is equal to the jumper's body weight.
An error of $10 \mathrm{~N}$ in selecting the bodyweight of the jumper produces an error in the flight height of $2-3 \mathrm{~cm}$.

The work-energy method is often the least reliable of the three methods. This method is subject to compounding of errors through the double integration process used to calculate the displacement of the jumper's c.m. The work-energy method is very sensitive to correct selection of an instant before the jump where the jumper is stationary and the ground reaction force is equal to the jumper's body weight. Examining the differences in accuracy between the three methods of calculating the flight height can be an illuminating exercise for the student.

\section{COUNTERMOVEMENT JUMP VERSUS SQUAT JUMP}

Most skilled jumpers can jump several centimeters higher in a countermovement jump than in a squat jump with the same vertical pushoff range. When comparing the force-time curves in Figs. 2(a) and 3(a), it is not obvious that the resultant impulse $\left(J_{\mathrm{GRF}}-J_{\mathrm{BW}}\right)$ for the countermovement jump is greater than that for the squat jump. Even when the two force-time traces are plotted on the same graph, the difference in the duration of the jumps makes it difficult ascertain which has the greater resultant impulse.

The superiority of the countermovement jump over the squat jump becomes readily apparent when comparing the force-displacement curves (and hence the work done by the jumper during the jump). Figure 6 shows the forcedisplacement curves corresponding to the countermovement jump and squat jump shown in Figs. 2 and 3. Figure 6 suggests that one advantage of a countermovement is that the leg muscles attain a higher level of activation and force before they start to shorten. In the squat jump, the force at the start of the upward phase of the jump (point $a$ ) is equal to the jumper's body weight. The muscles in the jumper's legs have only a low level of activation and force; just enough to maintain the body in a squat position. To start moving upwards, the jumper greatly increases the activation of the leg muscles, but it takes time (and distance) for the ground reaction force to build up to a high level.

In contrast, the ground reaction force at the start of the upward phase in the countermovement jump (point $d$ ) is already much greater than body weight. The levels of activation and force in the jumper's leg muscles are high because the jumper has to slow and then reverse the initial downward motion. The jumper thus performs more work early in the upward phase of the jump than in the squat jump, and so the jumper has a higher takeoff velocity and a greater flight height. In the jumps shown in Fig. 6, the jumper performed 27 $\mathrm{J}$ more work and jumped $4.5 \mathrm{~cm}$ higher in the countermovement jump than in the squat jump.

The mechanisms responsible for the enhancement of force in a countermovement jump are hotly disputed. Bobbert et al. ${ }^{9}$ and Ingen Schenau et al. ${ }^{10}$ argue that pre-stretching of the muscles allows the muscles to develop a higher level of active state and force before starting to shorten, as suggested above. However, some authors argue that the extra work is due to the release of elastic energy that has been stored in the muscles and tendons during the pre-stretch, and others explain the enhancement through 'potentiation' of the contractile proteins in the muscle, or through the contribution of the spinal reflexes. The interested reader is directed to a special issue of Journal of Applied Biomechanics (Vol. 13, no. 4, 1997) that 
was devoted to the mechanics and energetics of the stretchshorten cycle.

In a countermovement jump, the more vigorous the preliminary downward phase, the higher the jump. Figure 7 shows countermovement jumps with the same vertical pushoff range, but with different speeds in the downward phase. In these jumps, the greater the downward speed, the greater the force at the start of the upward phase, and hence the greater the work performed in the upward phase. This fact is known by most basketball and volleyball players. When jumping straight up for maximum height, the player performs a vigorous downward movement before leaping up off the ground. Even greater heights are achieved when the jumper uses a run-up into the jump. This is because the jumper is able to convert some of the kinetic energy generated in the run-up into gravitational potential energy. ${ }^{11}$

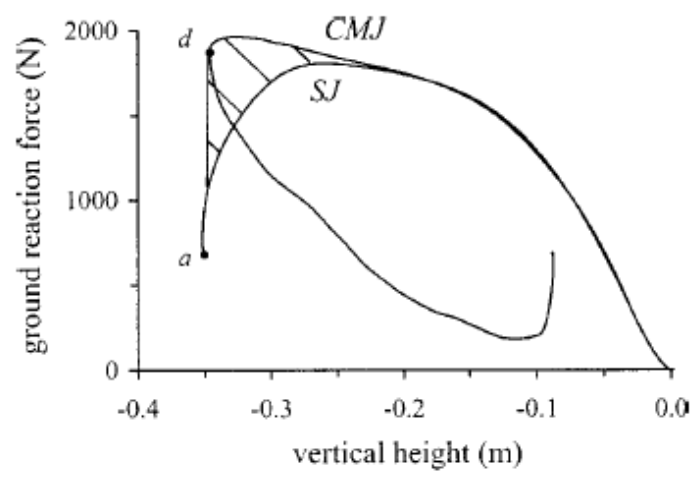

Fig. 6. Comparison of force-displacement curves for a countermovement jump (CMJ) and a squat jump (SJ). The shaded area indicates the greater work done by the jumper in the countermovement jump compared to the squat jump. (In both jumps, the vertical height $y$ $=0$ has been set to the height of the c.m. at the instant of takeoff.).

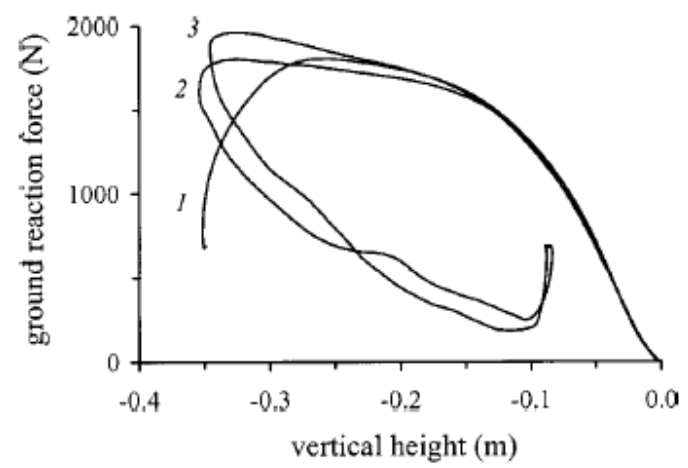

Fig. 7. Comparison of force-displacement curves for countermovement jumps with different speeds in the initial downward phase: (1) squat jump (no initial downward phase), (2) moderate downward speed, and (3) high downward speed.

\section{CONCLUDING REMARKS}

Although the physics of the vertical jump may be new to most physics educators, much of the material presented in this article is well known to teachers of human movement and sports biomechanics. ${ }^{12-14}$ Physics educators wishing to introduce a force platform into their classes would be well advised to seek the assistance of a biomechanics expert from within their university. Aspects of the present article that may be new even to a biomechanist are the curves of forcedisplacement and the application of the work-energy theorem to the vertical jump. Several companies manufacture force platforms with jump analysis software for use in research and in testing of athletes. For example, the QUATTRO JUMP package includes a large portable force platform and a software package that displays curves of force, velocity, height, and power. ${ }^{15}$ However, I do not know of any commercial software package that includes force-displacement curves. In my view, forcedisplacement curves have considerable potential in assessing the skill level of the jumper and in monitoring the effects of athletic training.

\section{ACKNOWLEDGMENTS}

Thanks to Ray Patton for helping to implement the JUMP ANALYSIS software, and to Rod Cross for comments on this manuscript.

\section{APPENDIX}

There are several key times and phases during a standing vertical jump. These are indicated on Figs. 2(a) $-2(e)$ by the letters $a$ to $h$.

$a$ : Point $a$ is the start of the jump. The jumper is initially standing upright and is stationary. The vertical height of the c.m. at this time has been set to zero.

$a-b$ : The jumper relaxes the leg and hip muscles, thus allowing the knees and hips to flex under the effects of gravity. The resultant force on the jumper $\left(F_{\mathrm{GRF}}-m g\right)$ becomes negative, and so the jumper's c.m. moves and accelerates downwards.

$b$ : Point $b$ marks the maximum downward acceleration of the jumper's c.m.

$b-c$ : The jumper's c.m. is still moving downward, but the jumper has started to increase the activation of the leg muscles and so the downward speed decreases. The resultant force on the jumper and the acceleration of the jumper's c.m. are still negative.

$c$ : At point $c$ the ground reaction force is equal to body weight, and so the resultant force on the jumper and the acceleration of the jumper's c.m. are zero. Point $c$ marks the maximum downward velocity. The region $a-c$ is sometimes called the 'unweighting' phase because the ground reaction force is less than body weight.

$c-d$ : The resultant force is now positive, and so the jumper is accelerating upwards, but still moving downwards.

$d$ : Point $d$ is the lowest point of the countermovement, where the jumper's c.m. is momentarily at rest (velocity is zero). The leg muscles are now strongly activated and the ground reaction force is close to maximum. A common error when examining the force-time curve is to identify point $b$ as the lowest point of the countermovement.

$d-e$ : This is the 'pushoff' phase, where the jumper moves upwards by extending the knees and hips. The velocity is now positive (upwards). For many jumpers, the maximum ground reaction force occurs early in the pushoff phase, shortly after the lowest point of the countermovement. 
$e$ : The ground reaction force has dropped to become equal to body weight. The resultant force on the jumper and the acceleration of the jumper's c.m. are therefore zero. Point $e$ marks the maximum upward velocity. The running high jump and long jump also display this phenomenon, where the maximum upward vertical velocity is not at the instant of takeoff, but at a short time before takeoff. ${ }^{16,17}$

$e-f$ : The ground reaction force drops below body weight and so the resultant force on the jumper and the acceleration of the jumper's c.m. are negative. The jumper's c.m. is still moving upwards, but it has started to slow due to the effect of gravity.

$f$ : Point $f$ is the instant of takeoff, where the ground reaction force first becomes zero. Note that the c.m. is higher at takeoff than at the start of the jump because the jumper has extended the ankle joints. (The jumper is standing on tip-toe.)

$f-g$ : The only force acting on the jumper is the jumper's weight and so the jumper is a projectile in free flight. The region $f-g$ marks the ascent of the flight phase, where the jumper's c.m. is moving upward but slowing down due to the effect of gravity.

$g$ : Point $g$ marks the peak of the jump, where the jumper's c.m. is momentarily at rest.

$g-h$ : This is the descent of the flight phase, where the jumper's c.m. is moving downward (velocity is negative) and speed is increasing.

$h$ : Point $h$ is the instant of landing, where the feet first contact the ground. The ground reaction force shows a sharp 'impact peak' and eventually becomes equal to body weight when the jumper is again standing motionless on the force platform (not shown).

In an article over 30 years ago in American Journal of Physics, Offenbacher ${ }^{18}$ presented curves obtained by Gerrish ${ }^{19}$ of force, velocity, height, and power as a function of time in a vertical jump. Offenbacher did not discuss these curves or indicate that he had made use of them in his undergraduate classes. Instead he merely stated that "the reader may find it interesting to analyze these curves in detail”. (How very true!) In Gerrish's study, the height of the c.m. of the jumper was obtained from a cinematographic analysis, rather than from the force platform data.

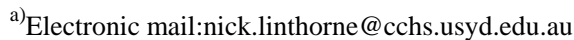

${ }^{1}$ R. Cross, "Standing, walking, running, and jumping on a force plate”, American Journal of Physics, 67 (4), 304-309 (1998).

${ }^{2}$ J. A. Major, W. A. Sands, J. R. McNeal, D. D. Paine, and R. Kipp, "Design, construction, and validation of a portable one-dimensional force platform”, Journal of Strength and Conditioning Research, 12 (1), 37-41 (1998).

${ }^{3}$ Our teaching laboratory is equipped with a Kistler force platform (type 9261A, with amplifier type 9803). Available from Kistler Instrument Corporation, Amherst, NY (www.kistler.com).

${ }^{4} \mathrm{P}$. V. Komi "Stretch-shortening cycle", in Strength and Power in Sport, edited by P.V. Komi, (Blackwell Science, Oxford, 1992), pp. 169-179.

${ }^{5} \mathrm{~A}$. Kibele, "Possibilities and limitations in the biomechanical analysis of countermovement jumps: a methodological study", Journal of Applied Biomechanics, 14 (1), 105-117 (1998).

${ }^{6}$ The most common method of measuring the height a person can jump is with the 'jump-and-reach' test. The jumper stands next to a wall with the arm stretched overhead and holding a piece of chalk. The jumper makes a mark on the wall, then performs a jump and makes another mark on the wall when at the peak of the jump. The difference between the two marks is the jump height. The presence of the wall and the requirement to make a mark on the wall restricts the performance of some jumpers.

${ }^{7}$ Another method of measuring the flight height is with a calibrated video analysis to monitor the motion of the jumper's c.m. Unfortunately, the jumper's c.m. is not at a fixed position within the body; its location changes in response to the bending of the legs, the position of the arms and the inclination of the trunk. In the 'segmentation' method, the location of the c.m. of the body is determined by summing the effects of the component segments of the body (head, trunk, limbs, etc). The mass and location of the c.m. of each of the body segments are estimated from data based on cadaver studies. The segmentation method is usually performed using computer-assisted digitising, calibration, and calculation techniques, and is relatively time-consuming. For further information see R. Bartlett, Introduction to Sports Biomechanics (E \& FN Spon, London, 1997), pp. 164-205.

${ }^{8}$ The flight time of a vertical jump may be measured using a 'contact mat' instead of a force platform. A contact mat is a thin sheet of foam rubber that is sandwiched by two layers of conducting foil. When the jumper is standing on the mat, the foam is compressed and the foil sheets are in contact, thus closing a timing switch. Available from Innervations, Muncie, IN (www.innervations.com) for about $\$ 600$.

${ }^{9}$ M. F. Bobbert, K. G. M. Gerritsen, M. C. A. Litjens, and A. J. van Soest, "Why is countermovement jump height greater than squat jump height?”, Medicine and Science in Sports and Exercise, 28 (11), 1402-1412 (1996)

${ }^{10}$ G. J. van Ingen Schenau, M. F. Bobbert, and A. de Haan, "Does elastic energy enhance work and efficiency in the stretch-shorten cycle?”, Journal of Applied Biomechanics, 13 (4), 389-415 (1997).

${ }^{11} \mathrm{R}$. McN. Alexander, "Optimum techniques for high and long jumps" Philosophical Transactions of the Royal Society of London: Series B, 329, 3-10 (1990).

${ }^{12}$ J. G. Dowling and L. Vamos, "Identification of kinetic and temporal factors related to vertical jump performance", Journal of Applied Biomechanics, 9 (4), 95-110 (1993).

${ }^{13} \mathrm{~J}$. Hamill and K. M. Knutzen, Biomechanical Basis of Human Movement (Williams \& Wilkins, Baltimore, 1995), pp. 476-478.

${ }^{14}$ E. A. Harman, M. T. Rosenstein, P. N. Frykman, R. M. Rosenstein, and W. J. Kraemer, "Estimation of human power output from vertical jump”, Journal of Applied Sport Science Research, 5 (3) 116-120 (1991).

${ }^{15}$ QUATTRO JUMP. Available from Kistler Instrument Corporation, Amherst, NY (www.kistler.com)

${ }^{16}$ J. Dapena and C. S. Chung, "Vertical and radial motions of the body during the take-off phase of high jumping”, Medicine and Science in Sports and Exercise, 20 (3), 290-302 (1988).

${ }^{17}$ A. Lees, P. Graham-Smith, and N. Fowler, "A biomechanical analysis of the last stride, touchdown, and takeoff characteristics of the men's long jump”, Journal of Applied Biomechanics, 10 (1) 61-78 (1994).

${ }^{18}$ E. L. Offenbacher "Physics of the vertical jump”, American Journal of Physics, 38 (7), 829-836 (1970).

${ }^{19}$ P. H. Gerrish “A dynamical analysis of the standing vertical jump”, Ph.D. thesis, Teachers College, Columbia University, 1934. 